\title{
RDUS
}

Revue de DROIT UNIVERSITÉ DE SHERBROOKE

Titre : $\quad$ LE DROIT AU SECOURS DANS LA PROVINCE DU QUÉBEC

Auteur(s): $\quad$ Alain KLOTZ

Revue: $\quad$ RDUS, 1990-1991, volume 21, numéro 2

Pages: $\quad$ 479-509

ISSN : $\quad 0317-9656$

Éditeur : Université de Sherbrooke. Faculté de droit.

URI : $\quad$ http://hdl.handle.net/11143/13444

DOI : https://doi.org/10.17118/11143/13444 
Page vide laissée intentionnellement. 


\section{LE DROIT AU SECOURS DANS LA PROVINCE DU QUÉBEC}

par Alain KLOTZ*

Fait unique en Amérique du Nord, la législature québécoise inscrit, en 1975, un droit au secours dans le chapitre des libertés et droits fondamentaux de la Charte des droits et libertés de la personne.

Dans ce qui suit, nous analysons en détail la portée générale de ce droit fondamental ainsi que l'obligation qu'il entraîne.

Nous soumettrons une façon d'harmoniser l'obligation de porter secours de l'article 2 de la Charte avec la législation existante, notamment avec l'article 1053 C.c..

Après étude des carences relevées dans l'obligation de porter secours, nous en proposons des amendements concrets.

Since 1975, the Quebec Charter of Rights and Freedoms contains a unique fact in North American legislation: the right to be rescued if one's life is in danger.

In what follows, we will provide a detailed analysis of the rights and duties deriving from this legislation.

We will submit a way of balancing out the obligation to bring succour (in article 2 of the Charter of rights) with the existing legislation, particularly the article 1053 C.c.

After close scrutiny of the shortcomings inventoried in this obligation to bring succour, we propose some concrete amendments.

*. $\quad$ Bachelier en droit et en rédaction de thèse de maîtrise en droit de la santé de la Faculté de droit de l'Université de Sherbrooke. L'auteur aimerait remercier, en premier lieu, monsieur le juge Jean-Louis Baudouin, alors professeur à l'Université de Montréal, pour ses encouragements et sans qui ce texte n'aurait pas vu le jour. Merci également aux professeurs André Morel de l'Université de Montréal et François Tôth de l'Université de Sherbrooke qui m'ont fait d'appréciables observations. 


\section{SOMMAIRE}

INTRODUCTION

I

ANALYSE DE L'ARTICLE 2 DE LA CHARTE . . . . 486

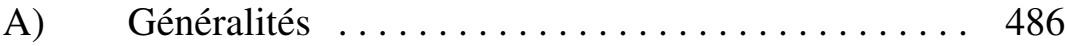

B) L'étendue de l'obligation de secours ......... 488

C) La limite à l'obligation de secours . . . . . . . 495

II UTILITÉ D'UN DROIT AU SECOURS . . . . . . . . . . 501

A) La faute d'omission contenue à l'article 1053 C.c. . . 501

B) Coexistence des articles 2 de la Charte et de 1053

C.c. ...................... 505

III CRITIQUES DE L'ARTICLE 2 ET CHANGEMENTS

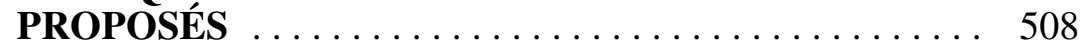

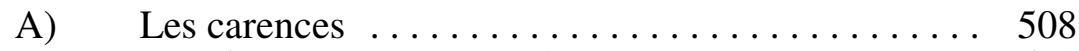

B) Les changements proposés $\ldots \ldots \ldots \ldots \ldots \ldots 514$

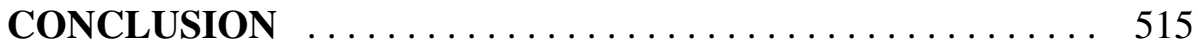




\section{INTRODUCTION}

Le droit au secours peut paraître une évidence à laquelle tout le monde convient de nos jours. Au Canada, le caractère sacré de la vie, enchassé dans les différentes chartes ${ }^{1}$, semble ancré dans l'esprit de tous. Même avant cela, la religion et la morale en ont toujours fait un commandement suprême. De plus, à l'intérieur des cadres professionnels, plusieurs lois et codes d'éthique imposent des obligations de secours et d'assistance en plus du respect de la vie et de l'individu ${ }^{2}$. Médecins, infirmiers et infirmières ou autres professionnels de la santé, tous sont sensibilisés au caractère sacré de la vie et aux obligations d'assistance et de protection qui en résultent.

Hormis la célèbre affaire St-Germain dans laquelle un généraliste de garde à l'urgence de l'hôpital Fleury avait refusé de traiter un patient cirrhotique amené par ambulance, vomissant du sang, sous prétexte d'un manque de lit disponible, les cas de médecins refusant de porter secours à un patient sont relativement rares dans la jurisprudence québécoise ${ }^{3}$.

Qu'en est-il en dehors du cadre professionnel? Des faits divers qui nous arrivent, certains révèlent une sordidité qui n'enlève pourtant rien à leur véracité. Ainsi, le 12 mars 1983, dans la ville de New Bedford, État du Massachussetts, une femme est violentée deux heures durant devant le patron et les clients d'un bar sans qu'aucun d'eux ne fasse quoi que ce soit pour secourir la victime. Les autorités ne furent même pas appelées sur les lieux ${ }^{4}$. Tous assistèrent apparemment passifs; on oserait même dire

1. $\quad$ Charte canadienne des droits et libertés, partie 1 de la Loi constitutionnelle de 1982 [annexe B de la Loi de 1982 sur le Canada (1982, R.-U, c. 11)], art. 7; Charte des droits et libertés de la personne, L.R.Q., c. C-12, art. 1.

2. L'article 23 du Code des professions, L.R.Q., c. C-26 prévoit la constitution de corporations professionnelles dans le but de protéger le public; l'article 4 de la Loi sur les services de santé et les services sociaux, L.R.Q.,c. S-5, assure à toute personne le droit aux services de santé et aux services sociaux; l'article 2.03.01 du Code de déontologie des médecins, R.R.Q., 1981, c. M-9, r.4, parle du respect de la vie, et l'article 2.03.47 du même code impose au médecin un devoir de secours envers son patient en danger; l'article 43 de la Loi sur la protection de la santé publique, L.R.Q., c. P-35, stipule une obligation à un établissement ou à un médecin de fournir des soins ou des traitements au patient dont la vie est en danger; l'article 2 de la Loi de la protection du malade mental, L.Q., 1972, c. 44, prévoit une aide au malade mental en danger; l'article 219 C.cr. criminalise l'omission à un devoir légal.

3. St-Germain c. R., [1976] C.A. 185, ici le refus de service et de secours constituait une infraction prévue à l'article 219 C.cr. Voir aussi, Services de santé et services sociaux-10, [1983] C.A.S., 851; Zuk c. Mihaly, J.E. 89-1307 (C.S.).

4. Diane KIESEL, «Who Saw This Happen?», (1983) 69 A.B.A. J. 1208. 
«moralement complices» si ce n'était que le terme complice n'est pas facilement acceptés.

La création d'un état d'urgence, mettant la vie d'un voisin en danger, oblige-t-elle à apporter l'assistance et le savoir faire nécessaires pour l'aider? Peut-on impunément regarder quelqu'un se noyer sans agir? Avons-nous le droit d'assister à l'agonie d'un blessé sans essayer de poser les premiers gestes de survie ou d'obtenir du secours? Dans ces cas, existe-t-il un droit au secours créant une obligation d'aide?

Jusqu'en 1975, sous l'influence de la Common Law, l'obligation de secours, au Canada, échappe à l'emprise du droit. En fait, le droit anglais ne reconnaît aucune obligation générale de secourir un étranger peu importe les circonstances $^{6}$. Sa devise est que le droit ne s'occupe pas de morale et, qu'à ce titre, chacun doit assurer sa propre sécurité et ne rien attendre de personne ${ }^{7}$. Le rôle du droit serait d'empêcher les gens de se nuire mais non de les encourager à s'entraider ${ }^{8}$. En fait, il s'agit d'un système basé sur un ensemble de valeurs doctrinales élevées au rang de conception économique prônant l'individualisme à l'extrême comme secret de la réussite ${ }^{9}$. Tout le monde serait libre d'agir comme bon lui semble quand il s'agit de secourir autrui même quand il s'agit de viol devant un public plus voyeur que passif ${ }^{10}$, de coups mortels donnés en présence de 38 témoins inactifs ${ }^{11}$, de noyades qui auraient pu être évitées par des moyens existants sur les lieux ${ }^{12}$.

5. En droit canadien, ne pas intervenir pour empêcher un crime, se comporter en spectateur passif n'est pas en soi une infraction à la loi. Voir: Dunlop et Stanley c. La Reine, [1979] 2 R.C.S. 881, 892; Dans Regina c. Salajko, [1970] 1 C.C.C. 352,353.(C.A.Ont.) la Cour d'appel de l'Ontario va plus loin: n'est pas considéré comme un encouragement actif le fait d'assister, pantalons baissés (sic), à un viol collectif. Tout au plus, pourrait-il s'agir d'un encouragement passif non répréhensible en droit criminel!

6. William L. PROSSER, Law of Torts, 4th Ed. St-Paul, West publishing Co, 1971, p. 340; Allen M. LINDEN, «Rescuers and Good Samaritans», (1971) 34 Modern Law Review 241.

7. Robert Justin LIPKIN, «Beyond Good Samaritans and Moral Monsters: An Individualistic Justification of The General Legal Duty to Rescue»,(1983-84) 31 UCLA L. Rev. 252, 293; Corothers c. Slobodian,(1973) 36 D.L.R.(3d) 597 (C.A. Sask.). Dans cet arrêt, la Cour d'appel de la Saskatchewan déboutait l'action en réparation intentée par une automobiliste blessée alors qu'elle tentait d'arrêter un camion afin de secourir les victimes d'un accident de la route dont elle venait d'être témoin.

8. A. L. GOODHART, «Rescue and Volontary Assumption of Risk», (1934-35) 5 Cambrige L.J. 192, 203.

9. Francis H. BOHLEN, «The Moral Duty to Aid Others as a Basis of Tort Liability», (1908) 56 U. Pa. L. Rev. 217.

10. Supra, note 5

11. L'affaire Genovese citée par D. Kiesel, loc. cit., note 4.

12. Beverly Anne SEAGRAVES, «The Duty to Rescue in California: A Legislative Solution?»,(1983-84) 15 Pacific L.J. 1261, 1265. 
Les tribunaux, aux États-Unis ${ }^{13}$, ainsi qu'au Canada ${ }^{14}$, n'ont pas accepté de sanctionner ces graves manquements à la règle la plus élémentaire de toute entité intelligente vivant en société: le secours. L'influence de la Common Law, bien que critiquée ${ }^{15}$, demeure forte aux États-Unis ${ }^{16}$ et au Canada ${ }^{17}$ sauf dans la province du Québec ${ }^{18}$.

À la fin du siècle dernier, les cours canadiennes de justice n'hésitaient pas à comparer le sauveteur occasionnel à un individu qui ne se mêlait pas de ses affaires, à un personnage offrant son propre sacrifice en s'exposant noblement et intentionnellement au danger qu'il avait recherché par sa conduite personnelle en tant que sauveteur négligent pour lui-même ${ }^{19}$. En fait, l'acceptation tacite des risques par le sauveteur est souvent alléguée pour lui refuser tout dédommagement.

Le concept du droit au secours n'est pourtant pas nouveau. L'ancienne Égypte et l'Inde en auraient fait une obligation voici quelques millénaires ${ }^{20}$. Certains pays ont très tôt légiféré sur la question ${ }^{21}$. Plus tard, d'autres États fédérés en ont fait autant en réaction à l'indifférence scandaleuse et révoltante du public devant les meurtres qui se produisaient sous leurs yeux ${ }^{22}$.

C'est en 1975 que le Québec décide de rompre avec la tradition britannique. La province va de l'avant à l'occasion d'un important

13. $I d$. voir aussi D. Kiesel, loc. cit., note 4.

14. Dunlop c. La Reine; R. c. Salajko, précités, note 5; R. J. LIPKIN, loc. cit., note 7, 291.

15. James Barr AMES, «Law and Morals», (1908) 22 Harv. L. Rev. 97; Sam B. WARNER, «Duty of a Railroad Company to Care for a Person it Has Without Fault Rendered Helpless», (1919) 7 Cal. Law Rev. 312; Warren A. SEAVEY, «I Am Not My Guest's Keeper», (1960) 13 Vand. L. Rev. 699; W. L. PROSSER, op.cit., note 6.

16. F. H. BOHLEN, loc. cit., note 9; W. L. PROSSER, op. cit., note 6. Malgré cette influence, certains états comme le Vermont et le Minnesota ont légiféré en faveur d'un droit général de secours: infra, note 22.

17. Corothers c. Slobodian, précité note 7. L'Alberta et La Nouvelle-Ecosse ont cependant adopté une loi pour le Bon Samaritain en 1969, sans pour autant créer une obligation spécifique de secours. Terre-Neuve leur emboite le pas en 1971, voir annexe p. 1 à 3.

18. Le 28 juin 1976, le droit au secours entrait en vigueur au Québec sous la forme d'une obligation d'assistance à personne en danger de mort, infra, note 38 .

19. Anderson c. Northern Railway of Canada, (1876), 25 U.C.C.P., 301, rapporté et commenté dans: Allen M. LINDEN, «Down with Foreseeability! of Thin Skulls and Rescuers», (1969) 47 Can. Bar Rev. 545, 560.

20. F.J.M. FELDBRUGGE, «Good and Bad Samaritans», (1965-66) 14 Am. J. Comp. L. 630.

21. Id., p. 635: dès 1845 , le Code criminel russe faisait état d'un droit au secours.

22. Par exemples, le Vermont en 1967 adopte un «Duty to Aid»: Duty to Aid the Endangered Act, Vt. Stat. Ann. Tit. 12, No. 519 (1967). Le Minnesota a amendé en 1983 un texte déjà existant pour créer un «Duty to Assist»: Good Samaritan Law, ch. 319, 1983 Minn. Sess. Law Serv. 2329. 
développement législatif dans le domaine des droits individuels et des libertés de la personne. Se distinguant nettement des législateurs des autres provinces canadiennes, le législateur québécois inscrit le droit au secours à l'article 2 de la Charte des droits et libertés de la personne le 27 juin $1975^{23}$, suivant ainsi, en partie, la recommandation que formulait, dès 1966, l'Office de révision du Code civil ${ }^{24}$.

Nous allons étudier en détail la portée générale de ce droit au secours ainsi que l'obligation qu'il entraîne pour tous.

Certaines critiques sont allées jusqu'à considérer l'article 2 de la Charte superflu puisqu'une faute d'omission est déjà incluse à l'article 1053 C.c. ${ }^{25}$. Nous analyserons ce point de vue.

Les faiblesses de l'article 2 de la Charte seront relevées et dans le souci d'une critique positive, nous en proposerons une nouvelle rédaction.

\section{ANALYSE DE L'ARTICLE 2 DE LA CHARTE}

\section{A) Généralités}

Nous aimerions tout d'abord rappeler que la Charte des droits et libertés de la personne, qui enchâsse le droit au secours à son article 2, s'inscrit dans une philosophie civiliste. Nous éviterons donc de faire référence ou d'emprunter des solutions à d'autres systèmes juridiques afin de mieux puiser dans notre droit civil. Celui-ci diffère de la Common Law par son aptitude à régler même des situations spécifiques, non prévues par le texte de la loi grâce à une rédaction générale et impersonnelle.

Dans l'étude qui suit, lorsqu'il a fallu donner une portée au texte de l'article 2 de la Charte québécoise, nous avons jugé plus juste de nous inspirer de la jurisprudence et de la doctrine françaises, abondantes dans le domaine de l'assistance à une personne en danger, puisque les principes de droit civil français nous sont connus. Le manque de doctrine et de

23. $\quad$ Charte des droits et libertés de la personne, précitée, note 1.

24. OFFICE DE RÉVISION DU CODE CIVIL, Rapport du comité des droits civils, Montréal, 1966, pp.13-14.

25. Francine DROUIN BARAKETT et Pierre-Gabriel JOBIN, «Une modeste loi du bon samaritain pour le Québec», (1976) 54 Can. Bar Rev. 290, 293 et s.. 
jurisprudence québécoises sur le droit au secours et l'obligation qui en découle ne nous laissait guère le choix.

Notre Cour d'appel respecte ce principe en ajoutant que le droit civil est suffisamment complet en lui-même pour n'avoir pas à emprunter des solutions aux systèmes de droit étrangers ${ }^{26}$. Nous utiliserons donc, à l'occasion, des réflexions, arguments ou jugements propres au droit français qui est le plus proche du nôtre. D'ailleurs, l'article 2 de la Charte ressemble étrangement à l'article $63 \mathrm{al} .2$ du Code pénal français qui impose une obligation de porter secours à une personne en danger ${ }^{27}$.

Rappelons enfin que dans notre système fédéral, c'est le gouvernement central qui a seul compétence en droit criminel ${ }^{28}$. Ainsi, en principe, le non-respect de l'obligation de porter secours ne peut entraîner autre chose que des recours civils à la charge de l'auteur de l'omission fautive $^{29}$. Cependant, la Charte s'est donné une certaine autonomie par rapport au droit commun en prévoyant à son article 49 des dommages exemplaires en cas d'atteinte illicite et intentionnelle à un droit qu'elle proclame, ou d'une violation délibérée à ses principes. De plus, par l'intermédiaire du Code criminel canadien $^{30}$, l'omission à une obligation imposée par une loi, tant fédérale que provinciale, peut entraîner, selon la nature de l'infraction, une négligence criminelle ${ }^{31}$. En d'autres termes, même si la province n'a pas voulu renforcer l'obligation de secours contenue dans l'article 2 de sa Charte en y prévoyant une sanction pénale spécifique par le truchement de l'article $87^{32}$, une telle sanction pourrait exister par le

26. Dodds c. Schierz, [1986] R.J.Q. 2623 (C.A.), voir particulièrement aux pages 26292630, M. le juge Monet fait des réserves quant à l'utilisation, en droit civil, des règles juridiques de la Common Law, même quand elles émanent de la Cour Suprême du Canada; Dans l'arrêt Chouinard c. Landry, [1987] R.J.Q. 1954, (C.A.), M. le juge Lebel abonde dans le même sens.

27. DALLOZ, Code pénal, Paris, Dalloz, 1989-90, Art. 63 al. 2: «quiconque s'abstient volontairement de porter à une personne en péril l'assistance que, sans risque pour lui ni pour les tiers il pouvait lui prêter, soit par son action personnelle, soit en provoquant du secours».

28. Loi constitutionnelle de 1867, 30 \& 31 Vict., R.- U., c.3, art. 91(27).

29. Au niveau de la peine encourue, le Québec se compare difficilement avec les pays unitaires comme la France qui eux, ont directement criminalisé l'omission de porter secours en prévoyant des peines de prison et des amendes dans la disposition constitutive de l'obligation elle-même. L'impact coercitif en est certainement plus grand que la simple possibilité de créer des infractions de type réglementaire aux conséquences pénales prévues à l'article 87 de la Charte québécoise.

30. Art. 219 C.Cr..

31. St-Germain c. $R$., précité, note $3 ;$ R. c. Fortier, infra, note 98.

32. L'article 87 prévoit un recours spécifique, en cas de contravention à certaines dispositions de la Charte, pouvant donner lieu à une condamnation suivant la Loi sur les poursuites sommaires, L.R.Q.,c. P-15. 
biais de la négligence criminelle prévue à l'article 219 C.cr. ${ }^{33}$. En fait, malgré ses faiblesses majeures, telles que nous les présenterons dans un chapitre ultérieur, nous remarquons qu'à l'inverse de certaines croyances ${ }^{34}$, l'article 2 n'est pas entièrement dépourvu de moyens coercitifs.

La Charte est quasi constitutionnelle ${ }^{35}$; elle occupe la première place au sommet de la hiérarchie des normes provinciales. Elle s'applique à tout le monde, gouvernement y compris ${ }^{36}$. En conséquence toute personne se trouvant sur le territoire de la province du Québec est assujettie à l'obligation de secours prévue à l'article 2 de la Charte. Cela comprend les étrangers comme les citoyens canadiens de naissance ou par naturalisation, ainsi que les Indiens, malgré la compétence fédérale sur ceux-ci ${ }^{37}$.

\section{B) L'étendue de l'obligation de secours}

L'article 2 prévoit que:

«Tout être humain dont la vie est en péril a droit au secours.

Toute personne doit porter secours à celui dont la vie est en péril, personnellement ou en obtenant du secours, en lui apportant l'aide physique nécessaire et immédiate, à moins d'un risque pour elle ou pour les tiers ou d'un autre motif raisonnable» ${ }^{38}$.(Les italiques sont de nous.)

La Charte crée une obligation de secours à la personne dont la vie est en péril. Dans ce cas, l'omission est un délit formel pourvu que la vie de la victime, et non son intégrité physique, ait été directement en péril! Ce n'est malheureusement qu'à cette condition que la victime aura un droit au secours ${ }^{39}$. Il doit évidemment s'agir d'un danger grave, aux conséquences mortelles ${ }^{40}$. Il est logique que l'abstention cesse d'être

33. R. c. Fortier, infra, note 98

34. QUÉBEC, ASSEMBLÉE NATIONALE, Journal des débats, 30ème législature, 2ème session, vol. $15, \mathrm{n}^{\circ} 79$, pp. 2741 et s. (12 novembre 1974).

35. Henri BRUN et GUY TREMBLAY, Droit constitutionnel, (supplément), Cowansville, Editions Yvon Blais Inc., 1985, p. 67.

36. Art. 54 de la Charte: «la Charte lie la Couronne».

37. H. BRUN, «La Charte des droits et libertés de la personne: domaine d'application», (1977) 37 Rev. du B. 179, 185-186; Loi constitutionnelle de 1867, art. 91(24).

38. Charte des droits et libertés de la personne, art. 2. Cet article n'entrait cependant en vigueur par proclamation que le 28 juin 1976.

39. Infra, «les carences de la loi».

40. Le déséquilibre potentiel d'un enfant dont la garde serait confiée à un père homosexuel en voie de changer de sexe, ne serait pas, selon nous, une cause suffisante pour alléguer le droit au secours, ceci étant dit avec respect à l'adresse d'un jugement rendu par le Tribunal de la Jeunesse: Protection de la jeunesse-169, [1985] T.J., 2011; Dans Gaudreault c. Drapeau, (1988) 45 C.C.L.T. 202, (C.S.) nous ne voyons pas de danger grave aux conséquences mortelles pouvant enclencher une obligation de secours dans 
délictuelle si la personne en danger a succombé avant qu'il n'ait été possible de lui porter secours: un mort n'est plus «celui dont la vie est en péril» que vise l'article 2 de la Charte; un mort n'est plus «une personne». En d'autres termes, le péril ne doit pas s'être déjà réalisé au moment où un tiers arrive sur les lieux et on ne pourrait reprocher à quiconque de ne pas secourir celui qui n'en a plus besoin, le danger auquel il était exposé s'étant pleinement réalisé. C'est la conclusion à laquelle arrive un tribunal français ${ }^{41}$ alors qu'il avait à juger le conducteur d'une automobile ayant pris la fuite après avoir écrasé un cycliste. La cour refusa de lui imputer le délit d'omission, étant donné que le cycliste avait été tué sur le coup et n'avait, de ce fait, plus besoin de secours. Rappelons cependant que, dans le contexte d'un accident de la route, au Québec, le nouveau Code de sécurité routière, entré en vigueur le 29 juin 1987, oblige le conducteur d'un véhicule routier impliqué dans un accident à rester sur les lieux et à fournir l'aide nécessaire à toute personne qui a subi un dommage ${ }^{42}$. Le Code de sécurité routière crée de toute évidence une obligation de secours supérieure à celle stipulée par la Charte $^{43}$.

Il nous semble que, dans l'appréciation de ce fameux péril à la vie, l'obligation de secours augmente quand il s'agit d'un médecin qui croise sur son chemin une personne en danger de mort, ou qui est appelé d'urgence sur les lieux d'un grave accident. Si l'omission de porter secours du simple passant sans connaissance médicale peut être due à son manque de perspicacité face à l'appréciation du péril ou de l'urgence de l'intervention, il ne pourrait en être de même quand cette omission émane de celui qui, de par sa profession, est certainement plus compétent pour poser un diagnostic et donner des soins efficaces ${ }^{44}$.

«Tout être humain dont la vie est en péril a droit au secours. Toute personne doit porter secours à celui dont la vie est en péril, personnellement ou en obtenant du secours, en lui apportant

la bagarre à mains nues que deux dames se livrent suite à une rixe familiale. Avec tout le respect qu'il se doit, la cour n'a-t-elle pas confondu le concept de personne en péril (intégrité physique) avec celui de vie en péril que requiert l'obligation de secours?

41. Trib. corr. Poitiers, 27 avril 1950, J.C.P. 1950.II.5618.

42. Article 168 du Code de la sécurité routière, L.R.Q., c. C-24.2 (sanctionné le 18 décembre 1986, entré en vigueur le 29 juin 1987, (1987) 119 G.O. 11, 3494).

43. Le Code criminel réprimande aussi le délit de fuite à l'article 252 .

44. Cass.crim., 31 mai 1949, J.C.P. 1949.II.4945 (Note J. MAGNOL); la position du médecin est plus critique quand la coutume est de se faire soigner à domicile. En France par exemple, un tiers des décisions publiées impliquent les médecins dans des poursuites pour omission de porter secours: Gilles LEVASSEUR 2ième Congrès de morale médicale, Paris, 1966, t.1, p.64, cité dans J. PENNEAU La responsabilité médicale, Paris, Ed. Sirey, 1977, p. 74, note 1. 
l'aide physique nécessaire et immédiate, à moins d'un risque pour elle ou pour les tiers ou d'un autre motif raisonnable» ${ }^{45}$. (Les italiques sont de nous.)

Par sa rédaction, nous pensons que l'article 2 impose une action directe au secouriste qui se doit d'agir d'abord en personne, quand cela est possible, ou en obtenant du secours. La conjonction «ou» dans le texte de l'article 2 n'ouvre pas, selon nous, une alternative évidente ou un choix sans préséance au devoir de secours. C'est le choix de la personne raisonnable qu'il faut retenir pour évaluer la conduite à suivre selon le cas. En effet, un sauveteur prudent et diligent qui constate un péril constant, précis et immédiat, n'aura guère d'option. Il devra, s'il le peut sans risque pour lui ou les tiers, agir par lui-même et vite, car s'il se borne à obtenir du secours au lieu de secourir concrètement, cela pourrait équivaloir à une omission de porter secours «en apportant l'aide physique nécessaire et immédiate» telle que stipulé dans la loi. Celui qui verrait un homme en train de s'immoler par le feu, devrait aussi agir immédiatement en essayant d'éteindre le feu au lieu d'aller obtenir du secours; il en est de même pour celui qui, pouvant transporter un blessé grave d'une région éloignée, se bornerait juste à prévenir les secours de la ville la plus proche au lieu d'agir dans le meilleur intérêt du blesséét.

Il est heureux qu'une telle option soit prévue, car dans certains cas d'accidents en milieu urbain, obtenir du secours serait préférable à une intervention maladroite. Le but est de secourir, non d'achever, celui dont la vie est en péril. Le but n'est pas non plus de prévenir un danger. Pour que naisse l'obligation, il faut l'existence préalable d'une situation donnant ouverture à un péril de mort imminent pour la personne à secourir: un état d'urgence certain. Un citoyen qui, voulant éviter que quelqu'un ne s'électrocute avec un câble électrique pendant au-dessus de la route, accrocherait un sac afin d'avertir les automobilistes du danger, serait plus imprudent que bon Samaritain, surtout si suite à cette manoeuvre, il s'électrocute lui même ${ }^{47}$.

Dans l'affaire Carignan c. Boudreau ${ }^{48}$, le juge Chevalier estime que, pour s'appliquer, le droit au secours requiert un état d'urgence qui n'existait pas dans le cas d'un enfant qui craignait de redescendre du toit sur lequel il était monté.

45. Charte des droits et libertés de la personne, précitée, note 1, art. 2.

46. Trib. corr. Bayeux, 22 juin 1954, D.1954.Jur.603-604.

47. Girard c. Hydro-Québec, J.E. 84-392 (C.S.) et [1987] R.L. 168 (C.A.).

48. [1987] R.L. 257 (C.A.), à la page 262. 
Par contre, l'état d'urgence existe lorsqu'une personne est victime d'un arrêt cardiaque; le médecin se trouvant sur les lieux ne peut refuser de porter secours sous aucun prétexte ${ }^{49}$.

Nous ferons état, dans un chapitre ultérieur, d'une faiblesse à l'obligation de secours qui ne prévoit que l'aide physique immédiate et non l'aide morale et préventive dans le cas d'un candidat au suicide par exemple $^{50}$. Nous voyons ici une volonté du législateur à vouloir régler des cas urgents créés par l'état de nécessité découlant d'un accident ou d'une catastrophe aussi imprévue que soudaine. C'est dans ce cadre bien précis que «l'aide physique nécessaire et immédiate» est à donner:

«Tout être humain dont la vie est en péril a droit au secours. Toute personne doit porter secours à celui dont la vie est en péril, personnellement ou en obtenant du secours, en lui apportant l'aide physique nécessaire et immédiate, à moins d'un risque pour elle ou pour les tiers ou d'un autre motif raisonnable» ${ }^{51}$. (Les italiques sont de nous.)

Il nous est difficile, en effet, d'attribuer une telle obligation d'aide physique, immédiate et sans risques dans le cadre d'une requête pour consultation de dossier ${ }^{52}$, d'une requête en vue d'obtenir l'autorisation de pratiquer une intervention chirurgicale ${ }^{53}$, d'une action en responsabilité d'un psychiatre à la suite du suicide de sa patiente ${ }^{54}$ ou dans le cadre de la garde d'un enfant par son père homosexuel ${ }^{55}$.

Il nous semble que dans ces trois jugements, le contexte n'est pas approprié à l'obligation de secours:

Dans l'affaire Goyette ${ }^{56}$, le juge accorde une requête pour autorisation de pratiquer une intervention chirurgicale sur une enfant de 26 mois atteinte

\footnotetext{
49. Zuk c. Mihaly, précité, note 3.

50. Infra, «les carences».

51. $\quad$ Charte des droits et libertés de la personne, précitée, note 1, art. 2.

52. $\quad$ Droit de la famille-140, [1984] T.J. 2049.

53. In Re enfant Maude Goyette et le Centre de Services Sociaux du Montréal Métropolitain, [1983] C.S. 429.

54. $\quad$ Cloutier c. C.H.U.L., [1986] R.J.Q. 615 (C.S.)

55. Dans le jugement Protection de la jeunesse-169, précité, note 40, il s'agissait de la capacité parentale d'un père homosexuel. Avec respect, il semble que le juge Rivet ait élargi les effets de l'article 2 de la Charte en incluant, faussement, le péril de l'intégrité physique ou morale: ici, une des conditions exigées par la loi faisait défaut, selon nous, à moins que le juge ne considère qu'un enfant est en danger de mort si la garde en est confiée à un parent homosexuel!?

56. Précité, note 53.
} 
de troubles cardiaques et du syndrome de Down. Les parents refusaient l'opération alléguant la qualité de vie future de l'enfant. À notre avis, il n'était pas nécessaire ni approprié de citer l'article 2 de la Charte à l'occasion de ce jugement; l'article premier stipulant le droit à la vie, l'article 30 C.c. protégeant l'intérêt de l'enfant, l'article 42 de la Loi sur la protection de la santé publique ${ }^{57}$ et l'article 38 de la Loi sur la protection de la jeunesse ${ }^{58}$ étaient suffisants pour décider du cas.

Dans Droit de la famille-140 ${ }^{59}$, il a été jugé que le droit à la vie et son corollaire, l'obligation de porter secours prévue à l'article 2 de la Charte, doivent avoir préséance sur le principe de la confidentialité dans les limites nécessaires pour sauver la vie d'une enfant adoptée et atteinte de leucémie. Dans cette affaire, il fallait absolument communiquer avec les parents naturels afin de tenter une greffe de moelle osseuse prise à même un frère ou une soeur de l'enfant. Avec respect, nous soumettons que l'article 2 de la Charte ne vise pas ce genre de situation, du moins quand d'autres dispositions y sont plus spécialement applicables. Or, dans le cas présent, l'article premier de la Charte et les articles 631 C.c.Q. et 30 C.c. étaient suffisants et plus appropriés que l'obligation générale de "bon samaritain" pour atteindre le but recherché. D'aucuns auraient pu prétendre, vu la hiérarchie des normes, que seul l'article 2 de la Charte pouvait contrer le principe fondamental du respect de la vie privée de l'article 5. Mais c'est oublier que les libertés et droits fondamentaux doivent s'exercer dans le respect du bien-être général des citoyens du Québec $^{60}$, et qu'à ce titre, les articles attitrés du Code civil ont préséance.

Enfin, dans l'affaire Cloutier $^{61}$, un psychiatre était poursuivi en dommages-intérêts à la suite du suicide de sa patiente. Encore là, le devoir du médecin devant le péril de mort de son client est énoncé et prévu dans les lois édictées, appropriées et spécifiques au contexte médical $^{62}$. Nous comprenons difficilement l'utilité de l'article 2 de la Charte dans un contexte professionnel aussi bien règlementé.

À propos des trois jugements ci-dessus, nous pensons qu'il est plus sage d'exercer les recours spécifiques existants avant d'invoquer le droit au secours prévu dans la Charte. Ce droit a été inséré dans un but

57. L.R.Q., c. P-35

58. L.R.Q., c. P-34.1.

59. Précité, note 52.

60. $\quad$ Charte des droits et libertés de la personne, précitée, note 1, art. 9.1.

61. Précitée, note 54.

62. Supra, «Introduction». 
autre; pour répondre à un besoin bien spécial et non pour remplacer ou écarter les articles déjà existants, lesquels sont appropriés aux situations apparaissant dans le domaine hospitalier ou dans un cadre professionnel.

Par sa formulation même:

«[...] en lui apportant l'aide physique nécessaire et immédiate à moins d'un risque pour elle ou pour les tiers». (Les italiques sont de nous.)

et par son historique, l'article 2 s'adresse d'abord et indéniablement à l'état de nécessité provoqué par une urgence en dehors du milieu hospitalier et pour régler les problèmes de civisme que pose le désintéressement du sort d'autrui, particularité de notre époque, et non aux situations décrites dans ces trois derniers jugements. Il reste qu'un visiteur ou toute autre personne non professionnelle de la santé, circulant dans un centre hospitalier seraient assujettis à l'obligation de secours avant toute chose puisque, dans ce cas, aucune loi spécifique ne s'applique à eux. D'un autre côté, si la jurisprudence et une certaine doctrine ${ }^{63}$ continuent à étendre l'obligation d'assistance de la Charte aux professionnels oeuvrant dans les services de santé, nous aurons alors la réponse à une des questions qui se posaient au premier symposium sur la médecine et le droit, tenu à l'Université de Montréal en 1975, au lendemain de l'adoption de la Charte des droits et libertés:

«Does the Bill extend existing obligations imposed on hospitals and medical personnel to render aid in emergency situations ${ }^{64}$ ?

Personnellement, il nous semble que ce serait dévaloriser l'esprit de la Charte que de l'alléguer dans des situations où la législation déjà existante suffit.

\section{C) La limite à l'obligation de secours}

Pour qu'une loi atteigne le but que s'est fixé le législateur, il faut qu'elle soit acceptable dans son application. Pour ce faire, il existe une dérogation à l'obligation de secours:

63. Sandra ROGERS MAGNET, «The Right to Emergency Medical Assistance in the Province of Quebec», (1980) 40 Rev. du B. 373; Alain BERNARDOT et Robert P. KOURI, La responsabilité civile médicale, Sherbrooke, Les Editions Revue de droit Université de Sherbrooke, 1980, nº 251, pp. 169-170.

64. Jean-Louis BAUDOUIN et William F. FOSTER, «La médecine et le droit: nouveaux aspects de la responsabilité civile médicale», (1975) 10 R.J.T. 6, 7. 
«Tout être humain dont la vie est en péril a droit au secours. Toute personne doit porter secours à celui dont la vie est en péril, personnellement ou en obtenant du secours, en lui apportant l'aide physique nécessaire et immédiate, à moins d'un risque pour elle ou pour les tiers ou d'un autre motif raisonnable» ${ }^{65}$. (Les italiques sont de nous.)

Malheureusement, ni la nature du risque envisagé par la loi pour se soustraire à l'obligation de secours, ni la valeur du «motif raisonnable», ne sont précisés, laissant ainsi aux tribunaux la tâche d'interpréter les dispositions pouvant excuser une omission de secours ${ }^{66}$. Cette dérogation est importante et permet de constater que personne n'est obligé à l'héroïsme; quant aux tiers, il ressort clairement de la loi qu'on a voulu les protéger des risques qu'ils pourraient encourir à l'occasion d'un sauvetage.

$\mathrm{Au}$ sujet du sauveteur, et afin de donner une certaine portée à l'obligation de secours, il nous semble que le risque encouru par lui doit être sérieux. L'appréciation de ce risque comporte quelques difficultés, cette notion étant différente d'un individu à un autre, eu égard à sa condition physique et ses antécédents. Le cascadeur professionnel pourra-t-il alléguer le risque que représentait pour lui le fait d'avoir à courir sur une étroite corniche suspendue à cinq mètres au-dessus du sol pour y déloger un couvreur qui, ayant glissé dans le vide, s'est retenu agrippé au bord de la corniche? Le juge devra-t-il introduire la considération du in concreto pour arriver à la conclusion que l'homme normal moyen a peur du vide et que cinq mètres représentent un risque sérieux alors que pour le cascadeur le risque est une notion qui ne se transige raisonnablement qu'au-dessus de 10 mètres?

Qu'en est-il du péril créé par la même personne qui est ensuite seule en état de porter secours? En d'autres termes, a-t-on le devoir de secourir la personne même que l'on vient de blesser de ses propres mains? Le remords suivant immédiatement le geste, porter secours à la victime mourante représenterait un risque de taille: celui d'être démasqué et poursuivi.

65. Charte des droits et libertés de la personne, précitée, note 1, art. 2.

66. Pour une étude approfondie sur les modes d'interprétation de la Charte québécoise, voir à ce sujet: Alain-François BISSON, «La Charte québécoise des droits et libertés de la personne et le dogme de l'interprétation spécifique des textes constitutionnels», (1986) 17 R.D.U.S. 18 
Quelle est la valeur du motif raisonnable que le législateur semble avoir voulu inclure à tout prix à l'article 2 in fine? S'agit-il de la norme de «l'homme raisonnable» (conformité au modèle de la personne raisonnablement prudente et diligente) de l'article 1053 C.c.? Si c'était le cas, l'obligation de secours s'en trouverait renforcée car, à bien y penser, il existerait peu de motifs permettant de se dégager de l'obligation de secourir une vie. S'agit-il d'un motif raisonnable d'un autre ordre, comme celui inclus dans l'ancien Code pénal allemand de 1870 à l'article 330 et repris dans le nouveau Code de 1975 à l'article 323 c) qui parle de «ne pas enfreindre d'autres devoirs importants» pendant le déroulement du secours ${ }^{67}$ ? Il pourrait aussi s'agir de motifs dégagés par la jurisprudence française à propos de l'obligation de secours du médecin qui n'est pas sur les lieux de l'accident. Pour éviter à ce dernier d'être souvent dérangé pour des choses insignifiantes, on lui reconnaît le droit d'apprécier, sous le seul contrôle de la connaissance des règles de l'art de sa profession exercée avec conscience, l'utilité, l'urgence et le bien-fondé de son intervention $^{68}$. Évidemment, cette prérogative n'existerait pas pour celui qui, appelé à l'aide, n'est pas médecin et ne saurait évaluer une situation de péril à la vie sur de simples indications transmises à distance.

La brutalité maladive d'un conjoint méchant et sadique serait-elle, un «motif raisonnable» pour ne pas appeler une ambulance alors que celui-ci s'est mortellement empoisonné par accident?

Le refus d'un établissement de recevoir un blessé grave, acheminé par ambulance, à cause d'un manque de ressources, constitue-t-il un «motif raisonnable» au sens de l'article 2 de la Charte?

Le risque de se faire poursuivre pour voies de fait à la suite de la pose d'un garrot salvateur sur la personne d'un blessé de la route qui, étant conscient, refusait d'être touché serait-il un motif raisonnable ${ }^{69}$ ?

67. La Cour d'appel a récemment dit que le fait de secourir une personne en détresse n'autorise personne à devenir son complice pour commettre un délit, en l'occurrence un méfait public: Ryel c. Procureur général du Québec, J.E. 90-232 (C.A.). La question reste entière quant à savoir si on peut commettre un méfait pour secourir une personne en danger de mort certain, sans être poursuivi.

68. Cass. crim., 31 mai 1949, D. 1949.Jur.347.

69. La personne humaine est inviolable: Art. 19 C.c.; Il est intéressant de remarquer que l'article 13 du Projet de loi 125, infra, note 124 stipule qu'aucun consentement n'est requis pour soigner une personne dont la vie ou l'intégrité physique sont menacés si un consentement ne peut être requis en temps utile. 
Le risque d'être poursuivi pour pratique illégale de la médecine représente-t-il un «motif raisonnable» pour ne pas poser un geste de survie considéré comme «acte médical» ${ }^{70}$ ? En effet, au cours de l'aide apportée à une personne dont la vie est en péril, il se peut qu'un geste dit «médical» soit porté afin de soulager ou sauver quelqu'un. L'éloignement de tout centre hospitalier et le facteur temps sont des incitatifs à poser de tels actes médicaux. Or, l'exercice de la médecine englobe un champ d'activités particulièrement vaste ${ }^{71}$ et va du diagnostic d'une simple fièvre à tout traitement ou suggestion de traitements possibles: pratiquer la respiration artificielle, aider à un accouchement, faire un simple massage, donner un sédatif, faire une injection ou un acte plus complexe mais connu du sauveteur...

Considérons un instant que ce sauveteur a fait ses études de médecine à l'étranger. L'Ordre des médecins ayant refusé de l'inscrire au tableau de l'Ordre, le dilemme serait de taille. Ce secouriste professionnel devra-t-il alléguer le «motif raisonnable» de l'article 2 de la Charte pour excuser son refus de poser un acte médical néanmoins connu de lui et seul secours valable dans le cas soumis ${ }^{72}$, ou enfreindre la Loi médicale car l'acte qu'il s'apprête à poser est moins illégal que l'abstention? Soit le moindre des deux maux, une nécessité, une obligation hautement morale qui en plus correspondrait à la notion de personne diligente de l'article 1053 C.c., la vie étant au-dessus de tout, la personne diligente agit; et la personne raisonnablement prudente, que faitelle? La question ne se posera plus si, un jour, la loi prévoit l'immunité du sauveteur ${ }^{73}$.

On pourrait aussi alléguer que le législateur n'a pas nuancé dans l'article 2 de la Charte comme le fait l'article 323(c) du Code pénal de la

70. Les médecins exercent une profession d'exercice exclusif: Code des professions, précité, note 2 , art. 31 et 32 .

71. En vertu de 31 al. 2 de la Loi médicale, L.R.Q., c. M-9, l'exercice de la médecine ne peut se faire que par des médecins dûment inscrits au tableau de l'Ordre des médecins tel que prévu à l'article 2 de la Loi médicale et ainsi que régi par le Code des professions, id.

72. Entre autres, donner un antidote, pratiquer une trachéotomie ou même fournir une pilule de nitroglycérine à la personne en état de crise cardiaque.

73. Infra, «Les changements proposés». Il est à noter que pour la première fois la Cour supérieure s'est indirectement penchée sur ce problème: Raîche c. Corporation professionnelle des médecins du Québec, [1989] R.J.Q. 1495, 1501 (C.S.). Il s'agissait d'un «naturo-thérapeute» poursuivi pour pratique illégale de la médecine. Le juge André Biron, dans une savante réflexion sur la portée des articles 31, 43 et 45 de la loi médicale, arrive à la conclusion que n'importe qui serait exempté des sanctions de la loi si, pour sauver une personne, il fallait poser un acte réservé aux médecins seuls. 
République fédérale d'Allemagne qui oblige le sauveteur à ne pas enfreindre «d'autres devoirs importants» pendant qu'il porte assistance. L'article 9.1 de la Charte pourrait-il avoir le même effet ${ }^{74}$ ?

Un accident fait naître un état d'urgence. C'est souvent une course contre la montre, il faut agir vite; le sauveteur potentiel a-t-il vraiment le choix ou le temps de peser le pour et le contre? Cet état d'urgence fait naître des prérogatives qui lui permettraient, selon nous, d'agir sans se préoccuper des conséquences légales des gestes que la situation l'obligerait à poser. L'homme raisonnable ne laisse pas mourir quelqu'un sous prétexte que l'acte de survie connu de lui est réservé aux médecins dûment inscrits au tableau de l'Ordre.

À propos de l'état d'urgence, le professeur Michel Vasseur de l'Université de Lille arrive à la conclusion suivante:

«[...] il est permis de se demander si toutes les règles de forme ne sont pas susceptibles d'être écartées en cas d'urgence ${ }^{75}$.

En France, l'urgence aurait donc une fonction d'éviction de tout ce qui fait obstacle à la réalisation rapide du résultat recherché.

Au Québec, l'urgence, en droit médical, apparaît comme revêtue d'une fonction dérogatoire à la règle de la loi ou du contrat, elle est source d'un droit d'exemption ${ }^{76}$. vite:

Aux É.-U., la notion d'urgence fait appel à la nécessité d'agir et

74. L'article 9.1 de la Charte rappelle que les droits fondamentaux doivent s'exercer dans le respect de l'ordre public et du bien-être général.

75. Michel VASSEUR, «Urgence et droit civil», (1954) 52 Rev. Trim. dr. civ. 405, 420 in fine.

76. Roger NERSON, «Urgence et droit médical», (1975) 10 R.J.T. 13. Code de déontologie des médecins, précité, note 2, art. 2.03.28; Voir aussi les articles 27, 34, 41 et 47 du Règlement sur l'organisation et l'administration des établissements, (1984) 116 G.O. II, 2745, qui traitent des dérogations que l'urgence et la nécessité apportent aux politiques d'admission élaborées par un conseil régional; A. BERNARDOT et R. P. KOURI, op. cit., note $63, \mathrm{n}^{\circ} 23$, p. $14, \mathrm{n}^{\circ} 207$, p. $137, \mathrm{n}^{\circ} 254$, p. 171; Projet de loi 125 , infra, note 124 , art. 13 . 
«The concept of emergency has been employed in the field of tort kaw to refer to an unforeseen combination of circumstances calling for spontaneous action to avoid an imminent danger» ${ }^{77}$.

Nous devons logiquement conclure que celui qui porte secours, remplissant de ce fait un devoir imposé par une loi quasi constitutionnelle, ne devrait pas avoir à s'inquiéter des règles de forme, ni de tout ce qui pourrait faire obstacle à la réalisation du sauvetage d'une vie.

Selon certaines critiques, l'article 2 serait une disposition superflue car superposée à une faute d'omission déjà élaborée par la jurisprudence à partir de l'article 1053 C.c. ${ }^{78}$. Nous vérifierons ce qu'il en est au juste et si vraiment un devoir général statutaire de secours est redondant, voire inutile en droit québécois.

\section{UTILITÉ D'UN DROIT AU SECOURS}

\section{A) La faute d'omission contenue à l'article 1053 C.c.}

L'article 1053 C.c. est certainement la disposition de droit civil la plus connue en tant que pilier de la responsabilité civile délictuelle. On a déjà dit à son sujet qu'il constituait à lui seul «une véritable Charte des droits» ${ }^{79}$. Nous sommes tous responsables pour les actes fautifs dommageables que nous posons si, ce faisant, nous sommes sains d'esprit. Quand on parle d'acte, on vise le fait actif, positif, définissable, descriptible: la commission, par opposition à l'abstention, à l'inaction.

En fait, lorsque la loi impose un devoir, nous sommes d'accord pour admettre la responsabilité en cas de manquement à ce devoir ${ }^{80}$, le contraire serait surprenant. Mais qu'en est-il de l'omission de poser un acte qui aurait pu empêcher la réalisation d'un dommage quelconque?

77. «Good Samaritans and Liability for Medical Malpractice», (1964) 64 Colum. L. Rev., $1301,1309$.

78. F. DROUIN BARAKETT et P.G. JOBIN, loc. cit., note 25, p. 293 et s.

79. Madeleine CARON, «Le Code civil québécois, instrument de protection des droits et libertés de la personne ?», (1978) 56 Can. Bar Rev. 196, 199.

80. M.A. SOURDAT, Traité de la Responsabilité, t.1, Paris, Editions Marchal et Billard, 1887, n. 442, p. 493. 
Nous savons que l'article 1053 C.c couvre les cas d'omission ${ }^{81}$, non pas l'omission à un devoir légal spécifique, comme le serait celle d'un médecin refusant de soigner un malade arrivé à l'urgence en ambulance ${ }^{82}$, mais bien l'omission au devoir général de se comporter en personne avisée, prudente et diligente selon la norme objective de la responsabilité civile.

Le fait de n'avoir pas porté secours à la personne dont l'intégrité morale et physique est en danger serait constitutif de faute selon les termes de l'article 1053 C.c. puisque, de l'avis du professeur André Nadeau, il ne fait aucun doute que les fautes d'abstention y sont implicitement incluses avec la même force que les autres fautes positives de commission. ${ }^{83}$ La faute d'omission consisterait à ne pas intervenir pour empêcher la réalisation d'un acte dommageable.

Cependant, le contraire est aussi soutenu, et l'omission à «aucun» devoir ou obligation spécifique d'agir selon la loi ne pourrait être fautive:

«C'est donc seulement lorsqu'il y a devoir pour une personne, [...] quand l'obligation lui est imposée par une loi [...], qu'il y a de sa part faute d'omission et responsabilité du fait dommageable ${ }^{84}$.

«[...] la faute suppose en effet [...] que le fait omis était ordonné par la loi» ${ }^{85}$.

À maintes reprises les tribunaux ont affirmé que l'omission ne peut entraîner de responsabilité civile tant qu'il n'y a pas, pour celui auquel on l'impute, l'obligation d'accomplir le fait omis. Ainsi, la Cour de cassation française a déjà dit:

«Une simple abstention ne saurait constituer un quasi-délit [...] lorsque le fait dont l'omission est relevée ne constitue pas une obligation civile» ${ }^{86}$.

\footnotetext{
81. Traité de droit civil du Québec, t.8, par André NADEAU, Montréal, Wilson et Lafleur, 1949, n ${ }^{\circ} 54$, pp. 35-36.

82. St-Germain c. R., précité, note 3.

83. A. NADEAU, op.cit., note 81, $\mathrm{n}^{\mathrm{0}}$ 55, pp. 36-37.

84. M.A. SOURDAT, op. cit., note 80.

85. P.-B. MIGNAULT, Le droit civil Canadien, t. 5, Montréal, Ed. Théoret, 1901, p. 333.

86. Cass. civ., 28 octobre 1935, D. 1935.Jur.537.
} 
Cette approche n'est pas étrangère à la Cour d'appel du Québec ${ }^{87}$. La Cour suprême du Canada a aussi eu l'occasion de se prononcer sur le sujet:

«Il me semble que cette obligation [celle d'alerter les pompiers] ne lui est imposée ni par la loi, ni par "les rapports nécessaires des hommes. [...] L'intimée n'avait pas par la loi l'obligation de faire ce qu'on lui reproche d'avoir omis. " ${ }^{88}$.

Dans ce dernier cas, la Cour est unanime à dire que la défenderesse (une compagnie fournissant un service de surveillance de gicleurs) n'avait pas l'obligation d'alerter les pompiers à la suite du début d'incendie éteint par les gicleurs qu'elle avait sous surveillance électronique. Avec respect, il nous semble que cela relève du domaine de la responsabilité délictuelle comme constitutif de faute par omission, cause d'un dommage aux termes de l'article 1053 C.c. Dans le cas présent, des dégâts importants avaient été occasionnés par l'écoulement de l'eau des gicleurs que seuls les pompiers pouvaient fermer s'ils avaient été appelés à temps ${ }^{89}$.

La faute est le fondement de la responsabilité civile, la clef de voûte du système: la responsabilité sans faute n'est pas prévue à l'article 1053 C.c. La faute consisterait justement dans cette abstention (par exemple de porter secours), contraire aux devoirs de prudence et de diligence. D'après le professeur Jean-Louis Baudouin, il n'est pas pour autant indispensable que ce devoir soit spécifique pour qu'il y ait faute:

«[...] l'obligation générale de se comporter en personne prudente et diligente contenue à l'article 1053 C.c. est, à notre avis, suffisante pour fonder une faute d'abstention, en l'absence d'une disposition légale ou réglementaire, touchant l'activité particulière que devait avoir le débiteur» ${ }^{90}$. (Les italiques sont de nous.)

87. Beauport (ville de) c. Laurentide Motel LTD. [1986] R.J.Q 981, 997, renversé par [1989] 1 R.C.S. 705.

88. Alliance Assurance Co. et Al. c. Dominion Electric Protection Co., [1970] R.C.S. 168, 171 et 173.

89. A ce sujet, il est intéressant de relever que le Code pénal norvégien, dans son obligation de secours, édicté à l'article 387(2), prévoit une obligation d'avertir à temps les autorités compétentes, de l'existence d'un incendie ou autres accidents analogues pouvant entraîner des risques pour la vie humaine.

90. J.-L. BAUDOUIN, La responsabilité civile délictuelle, Cowansville, Ed. Y. Blais, 1985 , n. 101, p. 61 et n. 18-22, pp. 12-15. 
Donc, même quand la loi n'impose aucun devoir spécifique d'agir, une responsabilité pour omission serait incluse à l'article 1053 C.c. car la responsabilité civile ne tient pas seulement à un fait positif volontaire, mais aussi à une simple imprudence et surtout à la négligence que n'aurait pas eu une personne raisonnable, prudente, diligente et soucieuse d'éviter un dommage.

Nous sommes donc d'accord avec une certaine doctrine ${ }^{91}$ pour laquelle une omission de porter secours à une personne en danger serait fautive eu égard à l'article 1053 C.c. par simple référence à la conduite de la personne raisonnable. Il est admis que le citoyen raisonnable et prudent doit prêter son secours ou prévoir l'imminence d'un danger. D'ailleurs, dans l'arrêt Moore ${ }^{92}$, la Cour suprême rapporte les propos du juge Bissonnette sur le devoir qu'aurait un commis de magasin de protéger les clients qui passaient près d'une flaque d'huile rendant le parterre glissant ${ }^{93}$.

La faute d'omission a été de nouveau alléguée très explicitement dans un arrêt de la Cour d'appel, confirmé cette fois en Cour suprême. Il s'agissait d'un chauffeur d'autobus qui refusait de reculer son véhicule sous lequel était prisonnier un enfant, parce qu'il avait reçu l'ordre de ne rien déplacer avant l'arrivée de la police:

«[...] il n'en résulte certes pas qu'un acte d'omission ne puisse être fautif quand celui qui s'abstient a, dans des circonstances normales, le devoir d'agir avec diligence et de se soucier de la vie d'autrui. Et ce devoir est d'autant plus impérieux que la faute d'omission empêcherait, si elle n'était pas commise, l'aggravation du préjudice causé à un tiers» ${ }^{94}$.

Plus récemment, un jugement de la Cour supérieure applique entièrement le principe de la faute par omission de l'article 1053 C.c. et, ce, même en l'absence de tout texte formel ou d'obligation spécifique ${ }^{95}$. Il s'agissait en l'occurrence d'un cas de harcèlement. L'employeur fut jugé

\footnotetext{
91. F. DROUIN BARAKETT et P.G. JOBIN, loc. cit., note 25, 295.

92. Eaton c. Moore, [1951] R.C.S. 470, 492.

93. Id., «[...] la connaissance et l'imminence d'un danger, lorsqu'il y a temps utile et moyen efficace pour y parer, constituent une faute pouvant engendrer responsabilité si l'on néglige ou s'abstient d'agir [...]».

94. Osborne c. Commission de Transport de Montréal, [1956] B.R. 853, 856, confirmé par [1958] R.C.S. 257.

95. Bussières c. Syndicat des Employés du Centre Hospitalier Robert Giffard et annexes, [1986], R.J.Q., 586 (C.S).
} 
négligent pour ne pas avoir agi compte tenu des plaintes de la victime. Une fois de plus, la faute par omission était reconnue comme incluse dans l'article 1053 C.c.

\section{B) Coexistence des articles 2 de la Charte et de 1053 C.c.}

La conclusion à laquelle certains juristes arrivent ${ }^{96}$ et selon laquelle l'adoption d'une loi spéciale (article 2 de la Charte), édictant une responsabilité civile, n'était pas nécessaire pour imposer un devoir général de secours vu l'existence de l'article 1053 C.c. nous paraît, avec respect, moins probante. Une critique basée sur un plan plus général tant politicosocial que juridique permet d'aboutir à une conclusion plus nuancée. Loin d'être inutile, le droit au secours tente officiellement de concilier morale et droit. C'est une nouveauté après toutes ces années d'influence du droit anglais ${ }^{97}$. La Charte édicte un droit au secours ainsi qu'une obligation générale de porter secours. Même si cette obligation est implicitement contenue à l'article 1053 C.c., elle n'est cependant pas décelable pour les personnes non averties. La démarche que nous avons suivie dans les pages précédentes reste très académique et il serait injuste de l'imposer aux citoyens qui, bien que censés connaître les lois, ne peuvent être astreints à ce casse-tête juridique basé sur une interprétation jurisprudentielle et doctrinale.

Le droit au secours constitue, quant à lui, une affirmation législative relativement précise. La violation intentionnelle et illicite de ce droit est clairement condamnable par une action en dommages exemplaires sous l'article 49 de la Charte. Par l'intermédiaire de l'article 219 C.cr., une poursuite pour négligence criminelle serait aussi possible dans certains cas $^{98}$.

96. F. DROUIN BARAKETT et P.G. JOBIN, loc. cit., note 25

97. Supra, «Introduction».

98. Supra, «l'étendue de l'obligation»; R. c. Fortier, C.S., Longueuil, $\mathrm{n}^{\circ}$ 500-01- 050-805, 17 novembre 1980, Claire B. Joncas. Ce jugement étant introuvable au greffe, nous avons dû nous référer à la COMMISSION DE RÉFORME DU DROIT DU CANADA, Droit pénal, l'omission, la négligence et la mise en danger, document de travail 46, Ottawa, Ministère des Approvisionnements et Services Canada, 1985, p. 19. Cette décision, prononcée sur le banc en Cour supérieure, est importante et aurait dû faire, selon nous, jurisprudence. En refusant de fournir à sa compagne mourante les choses nécessaires à la vie, un conjoint a été accusé de négligence criminelle causant la mort (219 (1)b) C.cr.) pour l'omission au devoir légal de venir en aide selon l'article 2 de la Charte des droits et libertés de la personne. Il fut néanmoins acquitté ayant plaidé la théorie de l'ignorance suite à sa déficience mentale. Au sujet de jugements importants non publiés: Albert MAYRAND, «Tentative de récupérer une partie de la jurisprudence occulte», (1972) 3 R.D.U.S., 79. 
Si on accepte le droit comme fait social ou «instrument de construction du tissu social ${ }^{99} \gg$, c'est aussi un instrument qui permet la fabrication politique de la norme qu'on veut implanter ${ }^{100}$. En légiférant, le parlement se devait de doser une norme juridique acceptable par toute la population afin d'aboutir à un résultat et ne pas rebuter les esprits tout en essayant de changer les habitudes de chacun.

Le but de l'obligation de porter secours qui découle du droit au secours est, avant tout, d'inciter les passants à s'arrêter lorsqu'ils sont témoins d'un accident afin d'aider les victimes. Inscrit sous forme de devoir, dans une Charte des droits et libertés qui se veut éducative ${ }^{101}$, le droit au secours énonce une nouvelle philosophie politique d'un aspect des droits de la personne longtemps négligé, le tout dans une volonté de recherche de la justice, de la paix et du bien être général ${ }^{102}$.

En fait, certaines critiques que nous formulerons à propos des carences de l'article $2^{103}$ sont toutes relatives et se confinent uniquement à une comparaison avec l'idéal de l'homme raisonnable de l'article 1053 C.c. C'est d'ailleurs pour faire échec à ces faiblesses qu'il faut lire les deux articles ensemble. Depuis la reconnaissance officielle d'un droit au secours, il faut, dans une optique civiliste, donner effet au droit nouveau et l'harmoniser avec les dispositions déjà existantes. Il s'agit là d'une confirmation d'un droit au secours inclus dans l'article 1053 C.c. en raison de l'existence d'une faute par omission non évidente à première vue. Les articles de la Charte et 1053 C.c. se complètent: l'article 2 énonce un principe, lui-même corollaire de l'article premier de la Charte: le droit à la vie; les dérivés de ce principe se retrouvent à l'article 1053 C.c.: l'homme raisonnable n'attend pas que la vie soit en danger pour agir. Le législateur semble avoir reconnu ce principe par la définition qu'il donne du terme «sauveteur» à l'article $1(\mathrm{~g})$ de la Loi visant à favoriser le civisme $e^{104}$.

99. Jacques-Yvan MORIN, «Une Charte des droits de l'homme pour le Québec», (1963) 9 McGill Law Journal, 273.

100. «Toute loi est animée par un but que le législateur compte réaliser». Propos du juge Dickson traitant de la constitutionnalité d'une loi dans l'arrêt R. c. Big M Drug Mart Ltd, [1985] 1 R.C.S. 295, 331.

101. QUEEBEC, ASSEMBLÉE NATIONALE, Journal des débats, op. cit., note 34, p. 2749.

102. Charte des droits et libertés de la personne, précitée, note 1, préambule al. 3 et 4.

103. Infra, «Critiques de l'article 2 et changements proposés».

104. L.R.Q., c. C-20 art. 1(g): Au Québec, la Loi visant à favoriser le civisme prévoit qu'un sauveteur est «celui qui, bénévolement, porte secours s'il a un motif rai- sonnable de croire que la vie ou l'intégrité physique d'une personne est en danger». (Les italiques sont de nous.) On remarquera qu'ici, le secours est reconnu dans le cas où l'intégrité physique seule est en danger, ce qui, comme nous l'avons vu précédemment, n'existe 
Il faut désormais lire l'article 1053 C.c. à travers les principes des articles 1 et 2 de la Charte car il est nécessaire qu'il y ait coexistence. L'article 2 ne vient pas répéter les principes reconnus à l'article 1053 C.c. ou les annuler ${ }^{105}$ mais bien les soutenir. C'est là le but d'une loi hiérarchisée par son insertion dans un texte quasi constitutionnel:

«[...] donner une vie morale et intellectuelle à l'ensemble de notre législation ${ }^{106}$.

\section{CRITIQUES DE L'ARTICLE 2 ET CHANGEMENTS PROPOSÉS}

\section{A) Les carences}

Nous sommes conscients que les limites du droit au secours dont nous allons faire état ont été voulues par le législateur. D'ailleurs, certains ont déjà écrit que le droit était l'expression formelle de la politique $^{107}$. Louis-Philippe Pigeon abonde dans le même sens ${ }^{108}$.

L'article 2 vise à enrayer l'indifférence, caractéristique de notre siècle, de ceux qui s'abstiennent de porter secours. Mais le législateur a cherché à minimiser l'impact de sa loi $^{109}$. En effet, par ses termes, cette dernière ne prévoit un devoir d'assistance qu'aux cas où la vie même de la victime est en péril ${ }^{110}$. Donc, si l'intégrité physique ou morale de quelqu'un est compromise, cela ne suffirait pas à engendrer une obligation de secours, contrairement aux conclusions de deux récents jugements ${ }^{111}$.

Il est à noter que l'Office de révision du Code civil avait, dès 1966, par une formulation différente, élargi l'obligation de secours au

pas quand il s'agit de l'obligation de secours de l'article 2 de la Charte. Voir aussi infra «Les carences».

105. F. DROUIN BARAKETT et P.G. JOBIN, loc. cit., note 25, 299.

106. QUÉBEC, ASSEMBLÉE NATIONALE, Journal des débats, op. cit., note 34, 2746.

107. Elmer A. DRIEDGER, The Composition of Legislation: Legislature forms and Precedents, 2nd edition, Department of Justice, Ottawa, 1976.

108. Louis-Philippe PIGEON, Rédaction et interprétation des lois, Les Publications du Québec, 1986.

109. F. DROUIN BARAKETT et P.G. JOBIN, loc. cit., note 25, 290.

110. «Tout être humain dont la vie est en péril a droit au secours [...]» 1ère phrase de l'article 2 de la Charte des droits et libertés, (Les italiques sont de nous), supra, «L'étendue de l'obligation de secours»; Girard c. Hydro-Québec, précité, note 47; Cloutier c. Hôpital C.H.U.L., précité, note 54.

111. Protection de la Jeunesse-169 et Gaudreault c. Drapeau, précités, note 40. 
péril de l'intégrité physique aussi bien qu'au péril de la vie ${ }^{112}$. En stipulant que toute personne en péril a droit au secours, l'Office de révision du Code Civil reprenait la formulation de l'article 63 al. $2 \mathrm{du}$ Code pénal français ${ }^{113}$. La différence est grande car, selon la formule actuelle, la brutalité, les voies de fait ou encore un viol public du genre de celui commis dans un bar de la ville de New Bedford ${ }^{114}$ n'obligeront pas les témoins à faire quelque chose pour aider la victime et aucune responsabilité civile ne pourra être retenue contre eux en vertu de l'article 2 de la Charte. Dans ces exemples, le droit au secours n'existerait donc «pas encore», sinon sous l'article 1053 C.c. ${ }^{115}$ puisque dans aucun de ces cas la vie de la victime n'était directement en danger. Tant que le risque encouru n'est que moral ou physique et non mortel à brève échéance, l'obligation de secours n'est pas encore engagée ${ }^{116}$.

Nous admettrons que cette conception dénote une certaine étroitesse de vue ${ }^{117}$. L'article 2 de la Charte ne fait pas référence à un degré de violence, mais cherche uniquement à préserver la vie.

«Tout être humain dont la vie est en péril a droit au secours».

112. OFFICE DE RÉVISION DU CODE CIVIL, op. cit., note 24, pp.13-14, art. 3: «Toute personne en péril a droit au secours. Nul ne peut,sans excuse raisonnable, refuser ou négliger de prêter secours à une personne en péril ou de lui procurer les soins immédiats nécessaires à la vie.»

113. Art. 63 al. 2: «[...] quiconque s'abstient volontairement de porter à une personne en péril l'assistance que, sans risque pour lui ni pour les tiers, il pouvait lui prêter, soit par son action personnelle soit en provoquant du secours.» (Les italiques sont de nous). Les tribunaux français, sans reconnaître la simple détresse comme déclencheur de l'obligation d'assistance à personne en danger, reconnaissent néanmoins l'atteinte à la santé ou le danger de lésions corporelles comme suffisant pourvu qu'il soit imminent, constant et accidentel, c'est à dire soudain, imprévu et imprévisible. L'état de péril à l'article 63 al.2 du Code pénal français fait référence à tout péril grave corporel; l'obligation de secours se trouve renforcée. Ainsi, en 1978, la Cour de Cassation a déclaré un directeur d'école coupable d'avoir omis de porter assistance à personne en péril pour ne pas avoir fait intervenir un médecin auprès d'un malade qui délirait depuis dix jours. Cette accusation n'aurait pas été possible au Québec vu les limites de l'obligation de secours car, bien que l'intégrité physique et morale du délirant était en péril, sa vie ne l'était pas de façon immédiate telle que le requiert l'article 2 de la Charte. En 1985, l'article 63 al.2 du Code pénal a été interprété de façon encore plus large. La 16ième Chambre correctionnelle de Paris a rendu un jugement digne de faire jurisprudence en rangeant l'aide au suicide par correspondance parmi les cas de non-assistance à personne en danger!!

114. D. KIESEL, loc. cit., note 4.

115. Selon nous, il existe toujours un devoir légal d'agir même lorsque la vie n'est pas en péril, voir supra, «La faute d'omission contenue à l'article 1053 C.c.»

116. S. RODGERS-MAGNET, The Right to Emergency Assistance in the Province of Quebec, mémoire de maîtrise, Montréal, Faculté de Droit, Université de Montréal, 1977, p. 24.

117. F. DROUIN BARAKETT et P.G. JOBIN, loc. cit., note 25, 299. 
C'est sur cette affirmation de la vie qui est en péril qu'on pourra facilement jouer pour décider de la non-application de la Charte ${ }^{118}$. La formulation actuelle de l'article 2 exigeant que la vie d'une victime soit en danger de mort pour créer une obligation de secours est, selon nous, malheureuse. En effet, qui peut avec certitude alléguer que, suite à un accident, la vie de quelqu'un est menacée; peut-être un médecin? En fait, l'appréciation du degré, de la précision et de la constance du danger sera laissée au potentiel secouriste d'occasion qui décidera de l'urgence ou non de son intervention.

En restreignant de la sorte l'obligation de secours autour du péril de la vie seulement, la loi permet, à celui qui a négligé de porter secours, de se disculper alors qu'il pouvait secourir sans risque pour lui ou pour les tiers; c'est un encouragement à minimiser la perception du danger de mort afin d'excuser la passivité. Selon quels paramètres le juge tranchera-t-il pour juger eu égard à la preuve offerte? Il aurait été si simple de rendre cette disposition facilement applicable en ne faisant référence qu'au péril de la personne humaine comme le stipule la Loi visant à favoriser le civisme $^{119}$ et comme le suggérait l'Office de révision du Code Civil ${ }^{120}$.

Le deuxième reproche que l'on peut formuler à l'endroit de cette loi est d'avoir omis l'obligation d'aide autre que physique ${ }^{121}$. Ainsi, le support moral, outil indispensable au témoin intervenant dans une tentative de suicide, n'est pas une obligation prévue dans la loi et pourtant il est indéniable que, dans ce cas, la vie du candidat au suicide est, dans l'immédiat, en danger.

L'article 2 de la Charte a été jugé et critiqué avec sévérité ${ }^{122}$, comme étant une disposition superflue, dangereuse et incomplète. Nous avons déjà répondu à ces critiques. Cependant, nous aimerions insister sur un aspect primordial qui manque également à cette loi: aucune immunité n'y est prévue pour les fautes possibles du sauveteur remplissant somme toute un devoir, une mission que la loi lui impose. N'est pas directement prévue non plus son indemnisation pour dépenses ou pertes subies dans l'assistance qu'il apporte à la victime. En ce sens, nous partageons, en partie, l'opinion émise ci-haut, à savoir que l'article 2 serait incomplet. En fait, il s'agit d'un manque qui risque, à lui seul, d'atténuer

118. Girard c. Hydro-Québec, précité, note 47.

119. Précitée, note 104.

120. Supra, note 112.

121. F. DROUIN BARAKETT et P.G. JOBIN, loc. cit., note 25, 301.

122. Id., p. 290 et s. 
l'effet principal recherché, soit celui d'enrayer le mal de l'indifférence caractérisant notre société quand il s'agit d'aider son prochain. Un des principaux motifs du refus de porter secours est justement la peur d'être ensuite impliqué dans des poursuites judiciaires ${ }^{123}$. Cette protection légale n'est pourtant pas inconnue du législateur qui a su prévoir une immunité contre les actions civiles dans certaines de ses lois ${ }^{124}$.

La Loi visant à favoriser le civisme ${ }^{125}$, bien qu'étant un pas en avant, ne prévoit pas non plus d'immunité pour le sauveteur. Cette législation en faveur du civisme a un caractère pratique. Elle permet au sauveteur, ou sa famille, d'être dédommagé pour préjudices subis en portant secours bénévolement ${ }^{126}$. L'article 15 prévoit même une récompense monétaire! Ces dispositions ne viennent en rien atténuer les reproches formulés précédemment au sujet de l'article 2 de la Charte; le fait qu'elles se trouvent dissimulées dans une autre loi moins connue qu'une charte $^{127}$, enlève beaucoup d'impact à l'obligation de secours. Les secouristes potentiels, en plus de penser aux risques de poursuites advenant la moindre faute de leur part envers la victime, auront en tête les préjudices qu'eux-même pourraient subir, sans jamais se douter, qu'à certaines conditions, ils pourraient être dédommagés pour pertes matérielles à l'aide d'une disposition législative pratiquement inconnue du grand public.

Notons que la jurisprudence québécoise renferme quelques rares décisions, qui, par le biais des règles classiques du droit civil, ont permis le dédommagement de bons samaritains médecins intervenus de leur propre initiative sur les lieux d'un accident ferroviaire ${ }^{128}$, ou encore ont dédommagé l'intervention volontaire d'une personne voulant arrêter un

123. R.J. GRAY et G.S. SHARPE, «Doctors, Samaritans and the Accident Victim», (1973) 11 Osgoode Hall L.J. 1, 22.

124. Voir par exemple la Loi sur la protection de la jeunesse, précitée, note 58, art. 43. Notons aussi que le Projet de loi 125, présenté devant l'Assemblée nationale, première session, trente-quatrième législature, par M. Gil Rémillard, Ministre de la justice, prévoit une immunité concernant la personne qui porte secours à autrui, à moins de faute lourde ou intentionnelle: P.L. 125, art. 1467.

125. Précitée, note 104.

126. Id. art. $1(\mathrm{~g})$ et 2.

127. Dans son numéro de février 1988, le magazine «Justice» fait état de la Loi visant à favoriser le civisme dans sa fiche «Droit social» No. 13, ce qui nous semble une bonne façon de faire connaître une loi, d'apparence modeste mais qui, en réalité, devient une contrepartie indispensable de l'obligation de secours, une sorte de complément.

128. Paquin c. Grand Trunk RY. Co.,(1896) 9 C.S. 336, le juge Larue a tranché le litige en reconnaissant que l'immixtion positive du demandeur était basée sur un principe reconnu dans notre droit civil soit la gestion d'affaires pouvant entraîner une action de in rem verso contre le défendeur qui a tiré avantage de cette immixtion. 
cheval emballé ${ }^{129}$. En 1921, il fût même décidé qu'aucune formalité légale n'était nécessaire pour l'indemnisation d'un médecin secouriste alors qu'il y avait nécessité urgente et absolue d'intervenir, vu l'ampleur d'une grave épidémie. La notion d'intérêt public soutenait cette décision $^{130}$.

Malgré ces jugements, nous pensons qu'une loi, voulant changer les habitudes d'indifférence et favoriser le secours entre humains, devrait inclure un mécanisme protégeant le sauveteur de toute tentative de lui faire assumer les risques d'une poursuite en responsabilité engendrée par le secours d'autrui. Cela encouragerait le respect de la loi. Cependant, certains ne voient pas l'utilité d'une loi spécifique concernant le bon samaritain $^{131}$. On compte sur la compréhension des juges qui, selon toute évidence, apprécieraient in abstracto la responsabilité du secouriste eu égard à la situation d'urgence à laquelle il a fait face, ceci selon la tradition civiliste qui veut que la faute s'évalue en tenant compte de toutes les circonstances de l'espèce. L'intervention légale et généreuse se doit d'être traitée avec égards:

«la responsabilité de celui qui entreprend de soigner dans une situation d'urgence sera certainement appréciée beaucoup moins sévèrement que dans une situation ordinaire» ${ }^{132}$.

L'immaturité de l'article 2 de la Charte, qui ne fait pas référence au péril de l'intégrité physique, et qui ne prévoit pas d'exonération de la responsabilité civile pour le sauveteur, se doit d'être corrigée. Trop de gens associent au secours d'autrui la possibilité d'une poursuite éventuelle. Dans un contexte de crainte, le respect d'une telle loi est voué à l'échec. Pour être fonctionnel et respecté, l'article 2 devrait être modifié. Ceci aurait pour effet de renforcer les valeurs qu'il tente de défendre.

129. Lortie c. Adelstein, (1914) 46 C.S. 543, la gestion d'affaires avait là encore servi de base au juge Charbonneau pour accorder au demandeur un dédommagement pour pertes subies en prenant soin des intérêts du défendeur.

130. $\quad$ Tremblay c. Ville de Baie St-Paul, (1921) 59 C.S. 498.

131. J.-L. BAUDOUIN, loc. cit. note 64, p. 46.

132. Id. 


\section{B) Les changements proposés}

Pour couvrir les cas où non seulement la vie, mais aussi l'intégrité physique d'une personne est en danger, il faudrait reprendre la recommandation de l'Office de révision du Code civil ${ }^{133}$ et modifier le second alinéa de l'article 2 de la Charte comme suit:

«Toute personne doit porter secours à celui ou celle qui est en péril».

Ainsi, le droit au secours couvrirait aussi les cas de personnes molestées.

Pour éviter que ne soit écartée la nécessité d'une aide morale immédiate dans le cas d'un candidat au suicide, il suffirait de supprimer le mot «physique» du deuxième paragraphe qui ce lirait comme suit:

«Toute personne doit porter secours à celui ou celle qui est en péril, personnellement ou en obtenant du secours, en lui apportant l'aide nécessaire et immédiate $[\ldots] »$.

Pour corriger le manque de protection du sauveteur contre des poursuites éventuelles, il faudrait rajouter à la fin de l'article 2 de la Charte une référence à la Loi visant à favoriser le civisme. Cette dernière serait aussi amendée afin d'y inclure une mention de non-responsabilité spécifiant cependant que, pour les médecins, infirmiers et infirmières, cette clause n'aurait d'effet que pour les actes accomplis hors cabinet privé ou en dehors d'un hôpital; dans tous les cas, la faute grossière serait exclue. ainsi:

L'amendement de la Loi visant à favoriser le civisme se lirait

«Dans tous les cas, celui qui, de bonne foi, a porté secours, ne peut être poursuivi en justice, ni en raison des actes accomplis immédiatement sur les lieux et hors le cabinet du médecin ou de l'hôpital ni pour la mort du secouru, sauf en cas de faute grossière».

Ainsi, corrigé et complété, l'article 2 de la Charte des droits et libertés de la personne se lirait ainsi:

133. Supra, note 112. 


\section{Art. 2.}

«Toute personne en péril a droit au secours.

Toute personne doit porter secours à celui ou celle qui est en péril, personnellement ou en obtenant du secours, en lui apportant l'aide nécessaire et immédiate, à moins d'un risque pour elle ou pour les tiers.

La Loi visant à favoriser le civisme s'applique au sauveteur dans tous les cas».

\section{CONCLUSION}

$\mathrm{Au}$ terme de cette étude critique, nous conclurons sur une note néanmoins positive.

Tout d'abord, nous devons constater et réaffirmer qu'il existe un droit au secours statutaire et quasi constitutionnel dans la province du Québec.

L'article 2 de la Charte est véritablement un nouveau contrat social, la pierre angulaire d'une obligation d'assistance que ni le Code civil, ni le Code criminel ne règlent de façon évidente. Il faut tenir pour acquis que cette obligation est désormais statutaire et que la transgression de cette dernière peut donner lieu à une poursuite civile à l'aide d'une action en dommages et à une poursuite pénale par le biais du Code crimine $^{134}$. Cela rend l'obligation de secours plus efficace que ne le serait une simple déclaration de principe.

Le droit au secours est une première au Canada. Le législateur québécois lui a en plus donné un caractère fondamental en lui conférant une supériorité hiérarchique ${ }^{135}$.

Même si l'article 2 de la Charte ne peut être considéré comme une véritable «Loi du Bon Samaritain», celui-ci n'étant pas adéquatement protégé contre les conséquences de ses maladresses, on se doit d'y voir une nette amélioration par rapport à la philosophie du droit anglo-saxon

134. Article 219.

135. J.-Y.MORIN, «La constitutionnalisation progressive de la Charte des droits et libertés de la personne», (1987) 21 R.J.T. 25, p. 32 et s. 
qui ne reconnaît pas encore un droit général de secours à la personne en danger de mort ${ }^{136}$.

La récente prise de conscience des gouvernements au mieux-être social passe nécessairement par une évolution constante de la solidarité humaine entre les individus formant la collectivité. L'individualisme poussé à l'extrême, indifférent et aveugle, semble, pour l'instant, passé de mode si l'on en croit le préambule de la Charte provinciale ${ }^{137}$

L'affirmation solennelle du devoir général de secours n'a peut-être pas eu l'écho auquel on s'attendait. Il reste, qu'avec le temps, la communauté juridique, formée aux principes énoncés dans la Charte, saura certainement mieux exploiter cette nouvelle philosophie des droits de la personne, surtout si on allie la norme de la personne raisonnable de l'article 1053 C.c à l'obligation de secours de l'article 2 de la Charte des droits et libertés de la personne.

Entre temps, cette obligation de secours commence à se faire connaître des citoyens qui ne devraient plus se sentir brimés par un devoir somme toute bien naturel.

En 1748, Montesquieu écrivait que les lois éducatrices étaient celles qui préparaient à être citoyen ${ }^{138}$. L'obligation de secourir son prochain, contenue dans la Charte, fera-t-elle de nous de meilleurs citoyens?

Enfin, puisque l'histoire nous enseigne que la progression des droits et libertés de la personne s'est faite, selon les évolutions de chaque époque, par ajouts, il serait souhaitable aujourd'hui de renforcer l'affirmation de principe de l'article 2 de la Charte en la corrigeant dans le sens que nous avons proposé afin de confirmer son rôle éducatif ${ }^{139}$ et ainsi rehausser le patrimoine législatif et social de la nation québécoise.

136. Supra, «Introduction».

137. $\quad$ Charte des droits et libertés de la personne, précitée, note 1, préambule al.3 et 4.

138. Charles-Louis De Secondat MONTESQUIEU, De l'esprit des lois, St-Armant, Édition Flammarion, 1979, p. 155.

139. QUÉBEC, ASSEMBLÉE NATIONALE, Journal des débats, op. cit., note 34, p. 2742 (discours du ministre de la Justice M. Jérôme Choquette). 